\title{
Multipositronic systems
}

\author{
Kálmán Varga * \\ Physics Division, Argonne National Laboratory, Argonne, Illinois 60439, USA
}

The stability and structure of systems comprising a negative ion and positrons are investigated by the stochastic variational method. It is shown that the $\mathrm{H}^{-}$and the $\mathrm{Li}^{-}$ions can bind not only one but two positrons. The binding energies of these double positronic atoms $\mathrm{E}\left(\mathrm{H}^{-}, \mathrm{e}^{+}, \mathrm{e}^{+}\right)=0.57 \mathrm{eV}$ and $\mathrm{E}\left(\mathrm{Li}^{-}, \mathrm{e}^{+}, \mathrm{e}^{+}\right)=0.15 \mathrm{eV}$ are somewhat smaller than those of their single positronic counterparts $(\mathrm{E}(\mathrm{HPs})=1.06 \mathrm{eV}$ and $\mathrm{E}(\mathrm{LiPs})=0.32 \mathrm{eV})$. We have also found that $\mathrm{a} \mathrm{Ps}^{-}, \mathrm{Ps}^{-}$and a proton form a bound system.

36.10.Dr, 31.15.Pf.,71.35.-y,73.20.Dx

The many-body problem is conceptually simple and well defined in atomic physics: indistinguishable fermions (electrons) interact via the Coulomb potential in the external Coulomb field of the atomic nuclei. The solution of this many-body problem is very difficult, because in addition to the direct interaction between the electrons, their indistinguishability brings an exchange correlation into effect. Despite the complexity enormous progress has been made in this field which has been rapidly developing ever since the birth of quantum mechanics. The calculations have been mainly focused on systems (atoms and molecules) where fast electronic motion takes place in the field of slowly moving heavy positive charges.

Much less is known about systems which contain positively and negatively charged particles of equal or nearly equal masses. The simplest examples of these systems are the Positronium ion $\left(e^{+}, e^{-}, e^{-}\right)$(predicted by Wheeler [1] in 1946, experimentally observed by Mills [2] in 1981), the $\mathrm{Ps}_{2}$ molecule $\left(e^{+}, e^{+}, e^{-}, e^{-}\right)$(predicted by Hylleraas and Ore [3] in 1947, not observed yet in nature), or the HPs molecule (indirectly observed, see [4]). These systems have been extensively studied by various theoretical methods in the last few years [5]. The existence of these small systems makes theorists curious as to whether (similarly to molecules) larger stable systems containing positrons can also be formed. One can ask whether a system of $m$ electrons $n$ positrons (for example an $\left(3 e^{-}, 3 e^{+}\right)$ system) is bound or whether a positron, a positronium, a $\mathrm{Ps}^{-}$ion or a $\mathrm{Ps}_{2}$ molecule can attach itself to an atom or molecule.

The theoretical description of such systems (let alone the prediction of their stability against autodissociation) is obviously very difficult. The difficulty can largely be attributed to the fact that the electron-electron and the electron-positron correlations are quite different due to the attraction and to absence of the Pauli-principle constraint in the latter case. The tiny binding energies of these loosely bound extended systems require highly ac- curate calculations.

Recent calculations have given the very surprising result that a positron can cling to a neutral atom [6]. The simplest such positronic atom is the $\mathrm{Lie}^{+}$. The complexity of the calculation of its small binding energy is best illustrated by the fact that many otherwise successful methods had failed to predict the existence of the bound state of this system [7],8]. These calculations show that the energy of the $\mathrm{Lie}^{+}$is lower than that of the $\mathrm{Li}$ atom but the energy was not below the $\mathrm{Li}^{+}+\mathrm{Ps}$ dissociation threshold. The first rigorous proof showing that the positron can attach itself to a $\mathrm{Li}$ atom was given by Ryzhikh and Mitroy [6] by using the stochastic variational method (SVM) [9, 10]. This finding has been later confirmed by different theoretical approaches 11 [13. Other atoms (e.g. $\mathrm{Be}, \mathrm{Na}, \mathrm{Mg}, \mathrm{Cu}, \mathrm{Zn}$ and $\mathrm{Ag}$ ) has also been found to be capable of binding a positron 14 16.

There is an other family of positronic atoms which are formed when positronium is attached to an atom. The possibility of the existence of such systems is more obvious: removing the positron leaves behind a negatively charged ion so one can understand how the positron becomes bound. The simplest example of such system is the HPs molecule which has been the subject of numerous theoretical investigations and has been experimen-

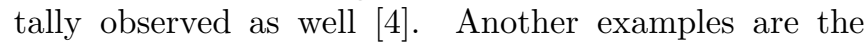
LiPs, NaPs and KPs atoms. The LiPs has been described by several microscopic methods [12, 14, while the other two bound systems have been predicted by a semimicroscopic model 15, 16].

In this paper we explore the possibility of the formation of stable atoms/ions containing two or more positrons. The simplest known example for such system is the $\mathrm{Ps}_{2}$ molecule. The study is inspired by the speculation that if a neutral atom can bind a positron then it may even be able to bind a positively charged $\mathrm{Ps}^{+}=\left(\mathrm{e}^{+}, \mathrm{e}^{+}, \mathrm{e}^{-}\right)$ion. This motivation can also be phrased in an other way: If positronium can bind itself to a neutral atom "A" forming a neutral system "APs" then can we attach a positron into APs?

The stochastic variational method systematically improves the correlation functions between the particles and it is especially suitable to solve Coulombic few-body problems. The method has been tested on a number of problems in different fields of physics and it has been proved to be highly accurate and reliable $\sqrt{10,17}$. The present study is restricted to states with total orbital angular momentum $\mathrm{L}=0$ and the following trial function is assumed

$$
\Psi=\mathcal{A}\left\{\mathrm{e}^{-\frac{1}{2} \mathbf{x} A \mathbf{x}} \chi_{S M_{S}}\right\}
$$


where $\mathbf{x}=\left(\mathbf{x}_{1}, \ldots \mathbf{x}_{N-1}\right)$ is a set of relative coordinates, $\chi_{S M_{S}}$ is the spin function, and $A$ is a matrix of nonlinear variational parameters. The nonlinear parameters are optimized by the stochastic variational method through a trial and error procedure. The details can be found in Ref. 110 .

This trial function includes explicit $\exp \left(-\alpha r_{i j}^{2}\right)$ correlation factors between the particles and it gives very accurate solutions provided that the nonlinear parameters (in the exponents) are properly optimized. As the number of parameters for a typical system is at least a few thousands a direct search for the optimal values is out of question. The stochastic variational method sets up a basis successively enlarging the model space by including the optimal trial functions. This basis was systematically improved by a refining procedure: The basis states were replaced by randomly chosen states which lower the energy. The energy found in this variational procedure converges to the upper bound of the exact ground state energy of the system. The Correlated Gaussians offer computational advantage: fast analytical evaluation of the matrix elements and good approximation to various wave functions. They also have well-kown drawbacks such as their slow convergence (compared to exponential functions) and the fact that they do not satisfy the cusp condition.

The simplest $\left(\mathrm{A}, \mathrm{Ps}^{+}\right)$is the $\left(\mathrm{H}, \mathrm{Ps}^{+}\right)=\left(\mathrm{p}, \mathrm{e}^{-}, \mathrm{e}^{-}, \mathrm{e}^{+}, \mathrm{e}^{+}\right)$ system. This five-body system can dissociate into $\mathrm{H}+\mathrm{Ps}^{+}, \mathrm{p}+\mathrm{Ps}_{2}$ or $\mathrm{HPs}+\mathrm{e}^{+}$, the lowest dissociation thresholds are shown in Fig. 1. To validate the method we have calculated the energies of the $\mathrm{Ps}_{2}$ and HPs molecules (see Table I.). The SVM significantly improved the theoretical values of the binding energies of these systems. Our calculation shows that the energy of the $\left(\mathrm{H}, \mathrm{Ps}^{+}\right)$is below the dissociation threshold and forms an electronically stable system. The $\mathrm{H}^{-}$ion can bind not only one but two positrons. The binding energy of $\left(\mathrm{H}, \mathrm{Ps}^{+}\right)=\mathrm{HPse}^{+}(0.021 \mathrm{a} . \mathrm{u})$ is comparable to that of HPs (0.039 a.u.). The convergence of the energy as a function of the basis dimension is shown in Table I.

The HPse ${ }^{+}$system can be also viewed as a bound system of a proton and a $\mathrm{Ps}_{2}$ molecule. The $\mathrm{Ps}_{2}$ molecule cannot bind an extra electron or positron because of the Pauli principle. Our calculations show that the $\mathrm{Ps}_{2}$ can bind a charged particle if it is distinguishable from the electron and the positron. The binding energy of a fivebody system $\mathrm{P}_{2}+\mathrm{x}^{+}=\left(\mathrm{e}^{+}, \mathrm{e}^{+}, \mathrm{e}^{-}, \mathrm{e}^{-}, \mathrm{x}^{+}\right)$consisting a hypotetical " $\mathrm{x}$ " particle is bound for any $0 \leq m_{e} / m_{x} \leq 1$ mass ratio. This has been checked by calculating the binding energy of that system for several different $m_{x}$ masses $\left(m_{x}=10^{50}, 100,10,8,6,4,2,1\right.$ in units of $\left.m_{e}\right)$. So while the $\left(\mathrm{e}^{+}, \mathrm{e}^{+}, \mathrm{e}^{+}, \mathrm{e}^{-}, \mathrm{e}^{-}\right)$is unbound the $\mathrm{Ps}_{2}$ can bind any charged particle, e.g. $\mu^{+}$or $\mathrm{p}^{+}$, because the Pauli principle does not restrict the motion of fifth particle in that case.

Some of the properties of these systems are shown in Table II. It is intriguing to compare the relative distances between the particles in HPs and HPse ${ }^{+}$. The electron-nucleus or electron-electron relative distances are almost the same in the two systems. The average nucleus-positron distance, however is substantially larger in $\mathrm{HPse}^{+}$. An other interesting property is that the relative distance between positrons is about twice that between electrons. All these facts suggest that a possible geometrical picture of the $\mathrm{HPse}^{+}$looks as an isosceles triangle formed by the two positrons and the proton and the two electrons are moving between the positive charges. The two positrons are placed on the vertices of the baseline of the triangle, and this baseline is so long that the system almost looks like as a linear chain. The HPse ${ }^{+}$ is somewhat related to $\mathrm{H}_{3}^{+}$. In $\mathrm{H}_{3}^{+}$three protons and two electrons form a very stable system, where the three protons are at the vertices of an equilateral triangle. By changing the mass of two of the positive charges this equilateral triangle is changed to an isosceles triangle, and in the positronic limit it looks like a linear chain.

The Li atom can bind a positron or a positronium forming an electronically stable $\mathrm{Lie}^{+}$or LiPs [6]. The binding energy of the $\mathrm{Lie}^{+}$is very small and it can be best viewed as a positronium orbiting around a $\mathrm{Li}^{+}$core. In our calculation we replace the positron with a $\mathrm{Ps}_{2}$ ion and try to determine the binding energy. In this case we have six active particles, four electrons and two positrons. This system has various different dissociation channels (see Fig. 2). The calculated energies of the relevant subsystems are listed in Table III.

Our calculation shows that the $\mathrm{Li}$ can bind a $\mathrm{Ps}^{+}$ion to form an electronically stable $\mathrm{LiPse}^{+}$. The calculated binding energy might not be very accurate due to the complexity of the system, but it is definitely below the lowest threshold (see Fig. 2). The convergence of the binding energy is shown in Table I. Further increase of the basis size would improve the ground state energy.

This system, again, can be viewed in different ways. One can say that a $\mathrm{Li}$ atom can bind a $\mathrm{Ps}^{+}$ion, or a $\mathrm{Li}^{-}$ ion is able to bind two positrons or the $\mathrm{Ps}_{2}$ molecule can attach itself to a $\mathrm{Li}^{+}$ion.

The relative distances between the particles in $\mathrm{LiPse}^{+}$ are shown in Table III. The average distance between the nucleus and the positron or between a positron and an electron is larger than that in LiPs but smaller than what one can find in $\mathrm{Lie}^{+}$. This would suggest a picture of $\mathrm{LiPse}^{+}$as a $\mathrm{Li}^{+}$core with an orbiting $\mathrm{Ps}_{2}$ molecule.

These systems are electronically stable but the positron electron pair can annihilate by emitting two photons. The annihilation rate is proportional to the probability of finding an electron and a positron at the same position in a spin singlet state (see eq. (21) in [15]). The expectation values of the positron-electron delta functions $\left(\delta_{e^{+} e^{-}}=\left\langle\Psi\left|\delta\left(\mathbf{r}_{e^{-}}-\mathbf{r}_{e^{+}}\right)\right| \Psi\right\rangle\right)$ are $1.4 \times 10^{-2}$, $1.1 \times 10^{-2}$ and $1.1 \times 10^{-2}$, for $\mathrm{Lie}^{+}, \mathrm{LiPs}$ and LiPse ${ }^{+}$. Due to the possible inaccuracy of the energy and wave function of the $\mathrm{LiPse}^{+}$system the annihilation rate should be considered as a qualitative estimate and it is about $\Gamma_{2 \gamma}=4.4 \times 10^{9} \mathrm{sec}^{-1}$.

The $\left(\mathrm{H}^{-}, \mathrm{e}^{+}, \mathrm{e}^{+}\right)$is a positively charged system so one 
may try to add one more electron to see if it remains stable. The convergence of the energy is shown in Fig. 3. The energy of the system slowly converges to the lowest (HPs+Ps) threshold and the size of the system continuously increases showing that this system is unlikely to be bound. Surprisingly, however, by adding two electrons to $\left(\mathrm{H}^{-}, \mathrm{e}^{+}, \mathrm{e}^{+}\right)$one gets a bound system as shown in Fig. 3. This system " $\mathrm{H}^{-} \mathrm{P}_{\mathrm{S}_{2}}$ " contains a proton, two positrons and four electrons, and can also be considered as a three-body system of a proton a $\mathrm{Ps}^{-}$and a $\mathrm{Ps}^{-}$ion as an analogy of the $\mathrm{H}^{-}$ion (where the electrons are replaced by the composite $\mathrm{Ps}^{-}$ions). The convergence of the energy is slow and the calculation of a more accurate binding energy would require a considerably larger basis dimension (see Table I.)

We have shown, for the first time, that neutral atoms can bind not only a single positron but a more complex positive charge, the $\mathrm{Ps}^{+}$ion as well. Besides the two cases $\left(\mathrm{HPse}^{+}\right.$and $\mathrm{LiPse}^{+}$) it is quite possible that other systems can be also bound. Although the investigation of larger systems is beyond the scope of the present method, other approaches (like QMC 12,18] or Fixed core SVM [15]) might be used to study the possible bound state of $\mathrm{Ps}^{+}$(or two positrons) with larger atoms/ions. Examples are (1) the recent QMC study of positronic water [18] and a new study with the Fixed core SVM which confirms the existence of the LiPse ${ }^{+}$and shows that a larger ion $\left(\mathrm{Na}^{+}\right)$ [20] can also bind a $\mathrm{Ps}_{2}$ molecule.

The investigation of these exotic systems are very important from theoretical point of view. These systems serve as test grounds for new methods: They provide a special environment where not only the electron-electron but other interleptonic correlations are also important.

While the chance of experimental observation of these systems is even more challenging than those of the positronic atoms [19], some of the properties of positronic systems can be affected by these bound states and the theoretical prediction of their existence might be very useful.

Systems, similar to $\left(\mathrm{p}^{+}, \mathrm{e}^{+}, \mathrm{e}^{+}, \mathrm{e}^{-}, \mathrm{e}^{-}\right)$might exist in semiconductors. Both the charged exciton (system of two electrons and a hole, akin to $\mathrm{Ps}^{-}$) and the biexciton (two electrons and two holes, similar to $\mathrm{Ps}_{2}$ ) have been experimentally observed [21,22]. Larger systems of "multiexcitons" (system of several electron-hole pairs) have also been observed [23 24]. These systems are of course different from the electron-positron systems because the electron-hole mass ratio $\left(\sigma=m_{e} / m_{h}\right)$ differs from unity and also because there is no annihilation so their observation might be easier. The stability for electrons and positrons indicates the stability for systems with slightly different mass ratios. The present study might give a hint for the existence of similar systems in semiconductors as well. In GaAs, for example, there are heavy holes $(\sigma=0.196)$ and light holes $(\sigma=0.707)$. A system similar to $\left(\mathrm{p}^{+}, \mathrm{e}^{+}, \mathrm{e}^{+}, \mathrm{e}^{-}, \mathrm{e}^{-}\right)$would comprise two electrons, a heavy, and two light holes.

This work was supported by the U. S. Department of
Energy, Nuclear Physics Division, under contract No. W31-109-ENG-39 and OTKA grant No. T029003 (Hungary).

[1] J. A. Wheeler, Ann. N. Y. Acad. Sci. 48, 219 (1946).

[2] A. P. Mills, Phys. Rev. Lett 46, 717 (1981).

[3] E. A. Hylleraas and A. Ore, Phys. Rev. 71, 493 (1947).

[4] D. M. Schrader, F. M. Jacobsen, N. P. Fradsen and U. Mikkelsen, Phys. Rev. Lett. 69, 57 (1992).

[5] For example see the references in D. B. Kinghorn and R. D. Poshusta, Phys. Rev. A 47, 3671 (1993), and in Y. K. Ho, Phys. Rev. A 48,4789 (1993).

[6] G. Ryzhikh and J. Mitroy, Phys. Rev. Lett. 794124 (1998).

[7] S.J. Ward, M. Horbatsch, R. P. McEachran and A. D. Stauffer, J. Phys. B: At.Mol. Opt. Phys. 223763 (1998).

[8] T. Yoshida and G. Miyako, Phys. Rev. A 544571 (1996).

[9] V. I. Kukulin and V. M. Krasnopolsky, J. Phys. G3, 795 (1977).

[10] Y. Suzuki and K. Varga, Stochastic variational approach to quantum mechanical few-body problems, SpringerVerlag, 1998.

[11] J. Yuan, B. D. Esry, T. Morishita and C. D. Lin, Phys. Rev. A58, R4 (1998).

[12] D. Bressanini, M. Mella and G. Morosi, J. Chem. Phys. 108, 4756 (1998).

[13] K. Strasburger and H. Chojnacki, J. Chem. Phys. 108 3218 (1998).

[14] G. Ryzhikh, J. Mitroy and K. Varga, J. Phys. B: At.Mol. Opt. Phys. 31 L265 (1998).

[15] G. Ryzhikh, J. Mitroy and K. Varga, J. Phys. B: At.Mol. Opt. Phys. 313965 (1998).

[16] G. Ryzhikh and J. Mitroy J. Phys. B: At.Mol. Opt. Phys. 321375 (1999).

[17] K. Varga, J. Usukura, and Y. Suzuki, Phys. Rev. Lett. 80, 1876 (1998).

[18] N. Jiang and D. M. Schrader, Phys.Rev.Lett 815113 (1998).

[19] J. Mitroy and G. Ryzhikh, J. Phys. B: At.Mol. Opt. Phys. 32 L111 (1999).

[20] J. Mitroy and G. Ryzhikh, J. Phys. B: At.Mol. Opt. Phys. 32 L621 (1999).

[21] G. Finkelstein, H. Shtrikman and I. Bar-Joseph, Phys. Rev. Lett. 74, 976 (1995).

[22] D. Birkedal, J. Singh, V. G. Lyssenko, J. Erland, and J. M. Hvam, Phys. Rev. Lett. 76, 672 (1996).

[23] A. G. Steele, W. G. McMullen, and M. L. W Thewalt, Phys. Rev. Lett. 592899 , (1987).

[24] M. Bayer et. al. Phys. Rev. B58 4740 (1998). 
TABLE I. The convergence of the total energy (E) and the energy relative to the lowest threshold $(\epsilon)$ as a function of basis size. The energy value in parenthesis (below the name of the system) is the lowest dissociation threshold. Atomic units are used. Infinite mass was used for the proton and $\mathrm{Li}$ nucleus.

\begin{tabular}{cccc}
\hline \hline system & Basis size & $\mathrm{E}$ & $\epsilon$ \\
HPse $^{+}$ & 100 & -0.809371 & 0.02017 \\
$(-0.78919)$ & 200 & -0.809993 & 0.02080 \\
& 400 & -0.810152 & 0.02096 \\
\hline LiPse $^{+}$ & 100 & -7.79502 & unbound \\
$(-7.7959)$ & 200 & -7.79811 & 0.00219 \\
& 400 & -7.80212 & 0.00620 \\
& 800 & -7.80510 & 0.00918 \\
\hline$\left(\mathrm{p}^{+}, 2 \mathrm{e}^{+}, 4 \mathrm{e}^{-}\right)$ & 100 & -1.02981 & unbound \\
$(-1.0512)$ & 200 & -1.04409 & unbound \\
& 400 & -1.05077 & unbound \\
& 800 & -1.05542 & 0.00423 \\
\hline \hline
\end{tabular}

TABLE II. Properties of Coulombic few-body systems. The particle " $\mathrm{x}$ " is distinguishable from both the electron and the positron, but is has the same mass as the electron. Atomic units are used.

\begin{tabular}{lcccccc}
\hline \hline system & Energy & $\left\langle r_{e^{-} p}^{2}\right\rangle$ & $\left\langle r_{e^{+} p}^{2}\right\rangle$ & $\left\langle r_{e^{-} e^{-}}^{2}\right\rangle$ & $\left\langle r_{e^{-} e^{+}}^{2}\right\rangle$ & $\left\langle r_{e^{+} e^{+}}^{2}\right\rangle$ \\
HPs & -0.78919 & 7.81 & 16.25 & 15.87 & 15.58 & \\
$\mathrm{Ps}_{2}$ & -0.51600 & & & 46.37 & 29.11 & 46.37 \\
$\mathrm{HPse}^{+}$ & -0.81015 & 7.49 & 31.84 & 15.14 & 33.71 & 65.40 \\
$\mathrm{x}^{+} \mathrm{Ps}_{2}$ & -0.55647 & 33.48 & 52.11 & 36.24 & 33.46 & 52.21 \\
\hline \hline
\end{tabular}

TABLE III. Total energies and expectation values of various operators in double positronic Li. Some other species are included for comparison. $r_{e^{+}}$and $r_{e^{-}}$are the distances between the nucleus and the positron and the nucleus and the electron, respectively. Atomic units are used.

\begin{tabular}{lcccccc}
\hline \hline system & Energy & $\left\langle r_{e^{+}}\right\rangle$ & $\left\langle r_{e^{-}}\right\rangle$ & $\left\langle r_{e^{-} e^{+}}\right\rangle$ & $\left\langle r_{e^{-} e^{-}}\right\rangle$ & $\left\langle r_{e^{+} e^{+}}\right\rangle$ \\
Lie $^{+}$ & -7.5323 & 10.03 & 3.44 & 7.83 & 6.43 & \\
$\mathrm{LiPs}^{2}$ & -7.7397 & 6.32 & 2.82 & 5.56 & 4.75 & \\
$\mathrm{LiPse}^{+}$ & -7.8051 & 7.51 & 3.30 & 6.12 & 5.71 & 6.15 \\
\hline \hline
\end{tabular}

FIG. 1. Energy levels of the HPse ${ }^{+}$and the HPs $+\mathrm{e}^{+}$and $\mathrm{H}+\mathrm{PS}^{+}$dissociation channels.

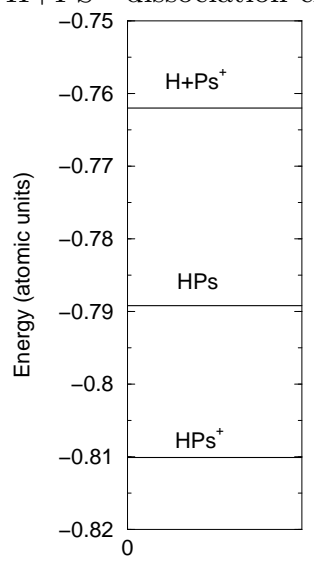

FIG. 2. Energy levels of $\mathrm{LiPse}^{+}$and its most relevant dissociation channels.

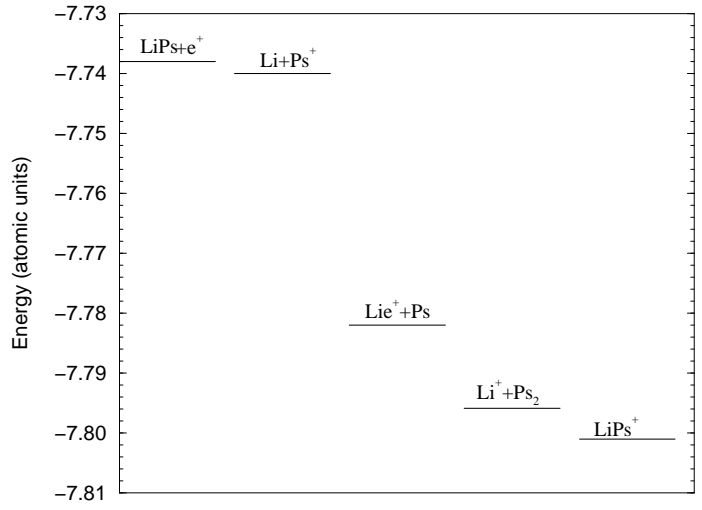

FIG. 3. Convergence of the energy of the $\left(\mathrm{p}^{+}, \mathrm{e}^{+}, \mathrm{e}^{+}, \mathrm{e}^{-}, \mathrm{e}^{-}, \mathrm{e}^{-}\right)$and $\left(\mathrm{p}^{+}, \mathrm{e}^{+}, \mathrm{e}^{+}, \mathrm{e}^{-}, \mathrm{e}^{-}, \mathrm{e}^{-}, \mathrm{e}^{-}\right)$systems. The dotted line is the HPs+Ps, the dashed line is the $\mathrm{H}^{-}+\mathrm{Ps}_{2}$, the long dashed line is the HPs+Ps ${ }^{-}$threshold.

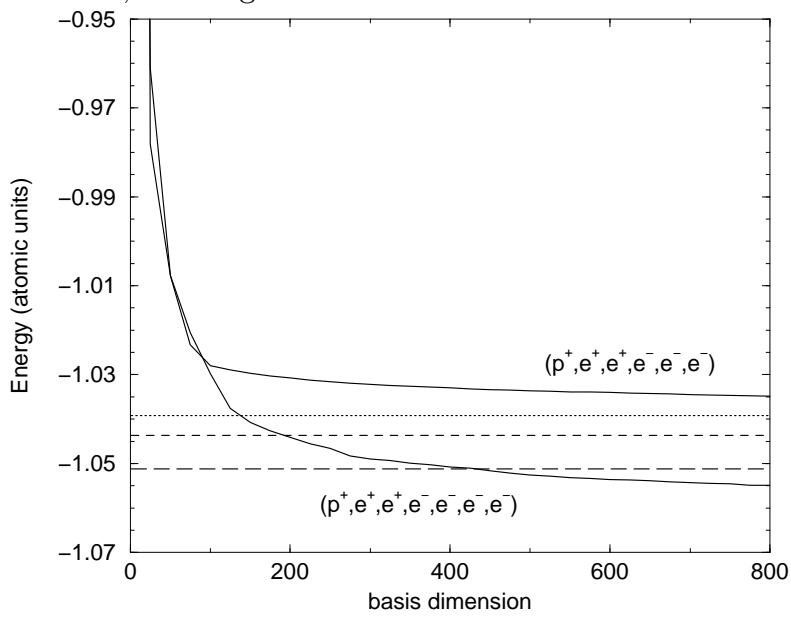

